\title{
Qualität und Struktur der ambulanten Versorgung von erwachsenen Patienten mit ADHS (Aufmerksamkeitsdefizit-) Hyperaktivitätsstörung)
}

\author{
Ergebnisse der RAABE-Studie [Retrospektive Daten-Analyse der ADHS-Behandlung \\ Erwachsener]
}

\section{Quality and Structure of Outpatient Care for Adults with ADHD (Attention Deficit Hyperactivity Disorder)}

\section{Results of the RAABE-Study [Retrospective Data Analysis of ADHD Treatment in Adults]}

\section{다 (i)}

\author{
Authors \\ Institute \\ 1 Klinik und Poliklinik für Psychiatrie und Psychotherapie, \\ Universitätsmedizin Rostock \\ 2 Kassenärztliche Vereinigung Bayerns \\ 3 Medice Arzneimittel Pütter GmbH \& Co. KG, Iserlohn \\ 4 Institut für Medizinische Informationsverarbeitung, \\ Biometrie und Epidemiologie, Ludwig-Maximilians- \\ Universität München
}

Johannes Thome ${ }^{1}$, Kerstin Behnke², Markus Gleitz ${ }^{3}$, Lydia Steer ${ }^{2}$, Martina Weiher ${ }^{2}$, Joerg Hasford ${ }^{4}$

Schlüsselwörter

Aufmerksamkeitsdefizit-/Hyperaktivitätsstörung, ADHS, Erwachsene, ambulante Versorgung

Keywords

attention deficit hyperactivity disorder, ADHD, adults, outpatient care

Bibliografie

DOI https://doi.org/10.1055/a-0880-1804

Online-Publikation: 13.8.2019 | Psychiat Prax 2019; 46:

317-323

(c) Georg Thieme Verlag KG Stuttgart · New York

ISSN 0303-4259

Korrespondenzadresse

Prof. Dr. med. Dr. phil. Johannes Thome, Klinik und

Poliklinik für Psychiatrie und Psychotherapie,

Universitätsmedizin Rostock, Gehlsheimer Straße 20,

18147 Rostock

johannes.thome@med.uni-rostock.de

\section{ZUSAMMENFASSUNG}

Ziel der Studie Darstellung der Versorgungsrealität erwachsener ADHS-Patienten in der ambulanten Versorgung. Methode Vollerhebung aller ambulanten GKV-Patienten $\geq 18$ Jahre mit ICD-Code F90.0 für das Jahr 2012 im Freistaat Bayern.

Ergebnisse Die Diagnoseprävalenz betrug 0,1\%. Circa 30\% der Patienten erhielten nach Diagnosestellung ein für die Behandlung der ADHS im Erwachsenenalter zugelassenes Arzneimittel. Drei Viertel der medikamentös versorgten Patienten bekam die Verordnung von einem Facharzt für ZNSErkrankungen. Rund $50 \%$ der Patienten erhielten auch psychotherapeutische Versorgung.

Schlussfolgerungen Da auch Hausärzte eine Rolle in der Versorgung von erwachsenen ADHS-Patienten spielen, sollte die Zusammenarbeit zwischen Fach- und Hausärzten sowie das Fortbildungsangebot verstärkt werden.

\section{ABSTRACT}

Objective Data on the quality and structure of outpatient care for adults with ADHD in Germany are scarce. The study describes the reality of care and identifies possible measures for improvement.

Method A complete survey of adults $\geq 18$ years of age with a diagnosis of ADHD (ICD-Code F90.0) covered by statutory health insurance was carried out in the outpatient setting in the German Free State of Bavaria in 2012.

Results The analysis revealed a diagnostic prevalence of ADHD in adults in Bavaria of $0.1 \%$, which was lower than expected based on ADHD prevalence estimates in the general population (about $3 \%$ ). Patients were diagnosed by specialists for CNS disorders and by general practitioners. About $30 \%$ of patients received a medication approved for the treatment of ADHD, and these were in approx. $75 \%$ of cases 
prescribed by specialists for CNS disorders. About $50 \%$ of the patients received psychotherapy.

Conclusion General practitioners play an important role for medical care of adult patients with ADHD. Continuous medical education programmes and collaboration between general practitioners and specialists is an urgent imperative for improving outpatient care of ADHD in adults.

\section{Einleitung}

Die ADHS (Aufmerksamkeitsdefizit-/Hyperaktivitätsstörung) ist eine durch Aufmerksamkeitsstörungen, Impulsivität, Überaktivität (bzw. innere Unruhe) und emotionale Labilität charakterisierte Entwicklungsstörung, die im Kindesalter beginnt und in bis zu zwei Drittel der Fälle bis ins Erwachsenenalter persistiert [1]. Das klinische Erscheinungsbild verändert sich typischerweise während der Lebensspanne. Betroffene berichten regelmäßig von erheblichen negativen psychosozialen Auswirkungen, die durch häufig auftretende Komorbiditäten wie affektive Störungen und Suchterkrankungen noch verschlimmert werden [2].

Während ADHS lange Zeit als Domäne der Kinder- und Jugendpsychiatrie galt, ist das klinische und wissenschaftliche Interesse an dieser psychischen Störung auch im Erwachsenenalter in den letzten beiden Dekaden erheblich gestiegen [3]. Es existieren effektive psychopharmakologische und psychotherapeutische Behandlungsoptionen [2,4] und es wurden eine Reihe nationaler und internationaler Leitlinien veröffentlicht $[5,6]$, um eine evidenzbasierte Versorgung dieser Patienten sicherzustellen.

Es wurde jedoch bislang kaum untersucht, inwieweit diese Empfehlungen in der Praxis umgesetzt werden. Es gibt Hinweise, dass die Defizite in der Versorgung von erwachsenen Patienten mit ADHS erheblich sind [7]. In den letzten Jahren wurden erste Studien zur Versorgungssituation veröffentlicht, die u.a. auf der Analyse großer staatlicher Gesundheitsregister z. B. in Skandinavien beruhen [8, 9].

Die vorliegende Untersuchung in Zusammenarbeit mit der KV Bayerns, die über Abrechnungs- und Arzneimitteldaten aller gesetzlich krankenversicherten Einwohner in Bayern verfügt (ca. 10,5 Mio., das entspricht ca. 85\% der Bevölkerung), gibt erstmals einen Überblick über die Versorgungsrealität erwachsener ADHS-Patienten in der ambulanten Versorgung durch niedergelassene Kassenärzte auf der Basis einer Vollerhebung für das Jahr 2012. Die deskriptiven Analysen lassen direkte Rückschlüsse auf mögliche Versorgungsdefizite und Schlussfolgerungen für eine Verbesserung der Versorgungssituation zu.

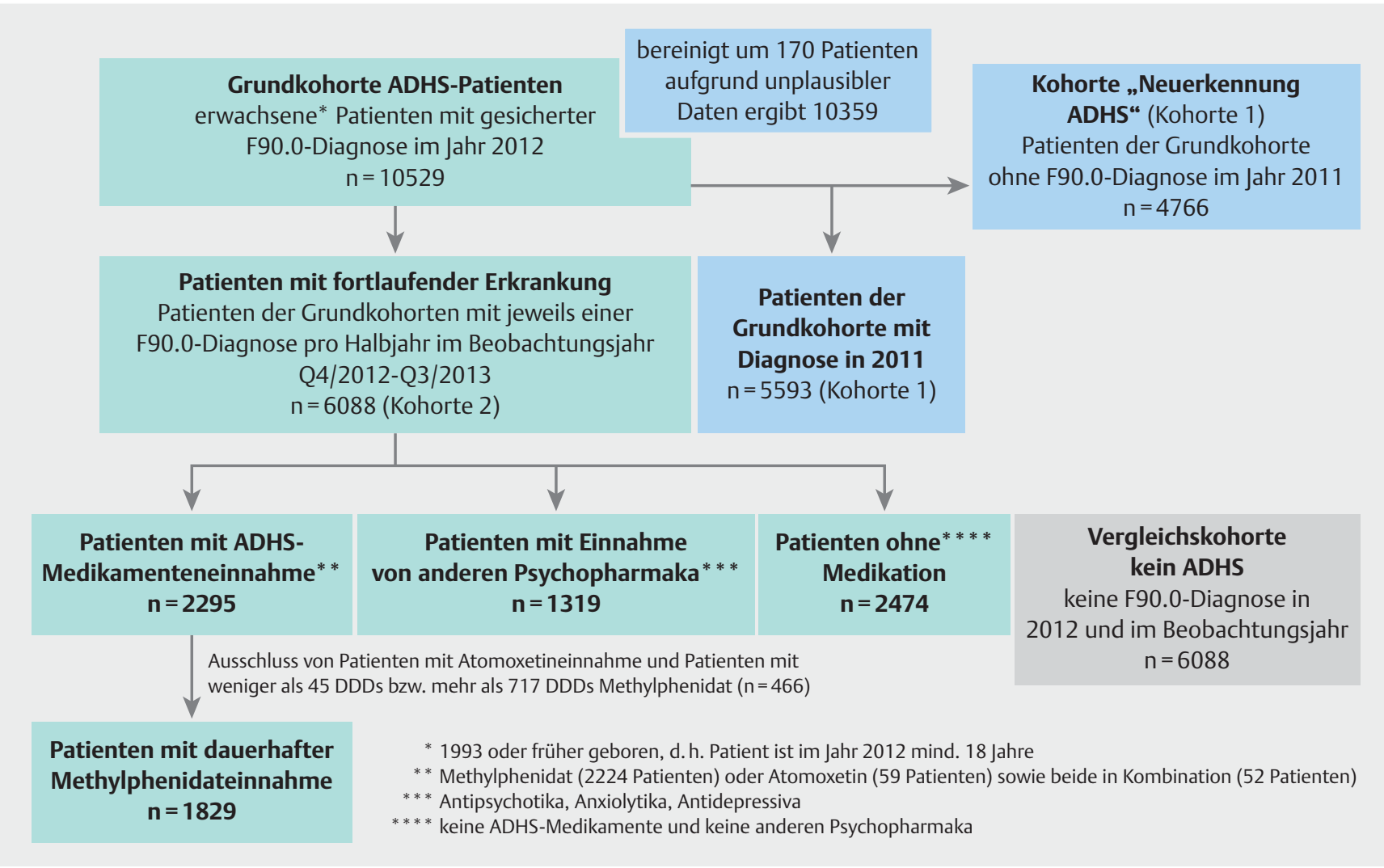

- Abb.1 Darstellung der Grundkohorte und Unterkohorten, mit denen die weiteren Analysen durchgeführt wurden. 


\section{Methode}

Als Datenbasis dienten die Routinedaten der Jahre 2009-2013 der Kassenärztlichen Vereinigung Bayerns sowie die bayerischen Arzneimittelverordnungsdaten des Zentralinstituts für die Kassenärztliche Versorgung in Deutschland, die über die pseudonymisierte Versichertennummer mit den Abrechnungsdaten zusammenführbar sind.

Einer Grundkohorte wurden alle gesetzlich versicherten, erwachsenen Patienten ( $\geq 18$ Jahre) zugeordnet, für die im Jahr 2012 zumindest einmalig die Diagnose einer gesicherten „Einfachen Aktivitäts- und Aufmerksamkeitsstörung“ (ICD-10-Diagnose F90.0; im Folgenden „ADHS-Diagnose“) im ambulanten Bereich dokumentiert wurde ( $\triangleright$ Abb. 1).

Patienten, die im Jahr 2011 keine ADHS-Diagnose aufwiesen, bildeten die Unterkohorte „Neuerkennung“. Untersuchungen zur Diagnosestellung und Behandlung dieser Patienten wurden über einen Zeitraum vom Quartal der Diagnosestellung (Indexquartal) und den darauffolgenden 3 Quartalen durchgeführt.

Aus der Grundkohorte wurde eine zweite Unterkohorte „Patienten mit fortlaufend dokumentierter Erkrankung“ gebildet. Für diese Patienten musste für den Beobachtungszeitraum (Quartal 4/2012 bis 3/2013) mindestens eine ADHS-Diagnose pro Halbjahr dokumentiert sein. Damit sollte sichergestellt werden, dass Patienten dieser Kohorte tatsächlich über einen längeren Zeitraum in Behandlung waren. Weiterhin wurde eine Vergleichskohorte gebildet, indem jedem ADHS-Patienten mit fortlaufender Erkrankung ein hinsichtlich Alter und Geschlecht „statistischer Zwilling“ ohne ADHS-Erkrankung aus der Grundgesamtheit aller bayerischen GKV-Patienten zugeordnet wurde.

Aus der Kohorte der „Patienten mit fortlaufend dokumentierter ADHS-Erkrankung “ wurden weitere Untergruppen gebildet: Patienten

- mit Einnahme von für die Behandlung der ADHS zugelassener Arzneimittel (Methylphenidat [MPH] oder Atomoxetin [ATX])

- mit Einnahme von anderen Psychopharmaka (Antipsychotika, ATC-Code N05A; Anxiolytika, ATC-Code N05B; Antidepressiva, ATC-Code N06A)

- ohne Einnahme von ADHS-Arzneimitteln bzw. ohne andere Psychopharmaka.

\section{Ergebnisse}

Im Jahr 2012 wurde im Freistaat Bayern für 10529 Patienten $\geq 18$ Jahre zumindest einmalig die Diagnose einer gesicherten „Einfachen Aktivitäts- und Aufmerksamkeitsstörung“ (ICD-10Diagnose F90.0) im ambulanten Bereich dokumentiert ( $\mathrm{Abb}$. 1). Bezogen auf den gesamten Datensatz (ca. 10,5 Mio. Patienten) entspricht dies einer Diagnoseprävalenz von ca. $0,1 \%$.

\section{Unterkohorte „Neuerkennung“}

Aus der Grundkohorte wurden 4766 Patienten identifiziert, für die im Jahr 2012 die Diagnose F90.0 vergeben wurde, im Jahr 2011 jedoch nicht. Eine weiterführende Analyse ergab, dass
- Tab. 1 Fachgruppen, die die initiale ADHS-Diagnose F90.0 codiert haben.

\begin{tabular}{|l|c|c|}
\hline Fachgruppe & $\begin{array}{l}\text { Anzahl } \\
\text { Patienten }\end{array}$ & $\begin{array}{l}\text { Anteil } \\
\text { Patienten (\%) }\end{array}$ \\
\hline Allgemeinärzte & 2114 & 49,06 \\
\hline Nervenärzte & 916 & 21,26 \\
\hline Psychiater & 304 & 7,06 \\
\hline hausärztliche Internisten & 297 & 6,89 \\
\hline Kinder- und jugendpsychiater & 263 & 6,10 \\
\hline Neurologen & 81 & 1,88 \\
\hline psychologische Psychothera- & 64 & 1,49 \\
\hline peuten - Verhaltenstherapie & & 1,44 \\
\hline Frauenärzte & 62 & 1,30 \\
\hline psychotherapeutisch tätige Ärzte & 56 & 3,53 \\
\hline sonstige Fachgruppen & 152 & 100,00 \\
\hline Summe & 4309 & \\
\hline
\end{tabular}

bei 1603 Patienten (ca. 34\%) bereits in den Jahren 2009/2010 eine F90.0-Diagnose vergeben worden war. Da jedoch auch bei diesen Patienten zumindest ein diagnosefreies Jahr vorlag, wurde die Zuordnung zur Unterkohorte „Neuerkennung“ beibehalten. Dabei ist der Begriff der „Neuerkennung“ nicht gleichzusetzen mit „Erstdiagnose“ oder gar der - laut aktuell gültiger klinischer Kriterien (noch) nicht zulässigen - Diagnose einer late-onset-ADHS ohne Symptome im Kindesalter. Da es in der vorliegenden Studie um die Analyse der Versorgungssituation ging, musste hier erfasst werden, wenn ein niedergelassener Arzt „neu“ eine ADHS erkannte (z.B. nach Arztwechsel durch den Patienten), unabhängig davon, ob zuvor (z. B. durch einen anderen Kollegen) bereits eine solche Diagnose gestellt worden war.

Bei mehr als der Hälfte aller neu diagnostizierten erwachsenen ADHS-Patienten (ca. 56\%) wurde die Diagnose von Hausärzten (Allgemeinärzte und hausärztliche Internisten) gestellt ( $\triangleright$ Tab. 1). Bei 457 Patienten wurde die Diagnose im Quartal der Diagnosestellung von mehreren Arztgruppen codiert, diese Patienten wurden in der Analyse nicht berücksichtigt.

Innerhalb eines Jahres nach der Diagnosestellung hatten 710 der durch Hausärzte diagnostizierten Patienten (ca. 29\%) einen Facharzt für Erkrankungen des ZNS aufgesucht. Bei 142 dieser Patienten wurde die ADHS-Diagnose bestätigt, bei 568 Patienten stand eine andere Erkrankung überwiegend aus dem Bereich der affektiven Störungen sowie der Angst- bzw. Belastungsstörungen im Vordergrund.

Nach der Diagnosestellung erhielten 1429 Patienten (ca. $30 \%)$ der Unterkohorte „Neuerkennung“ ein für die Behandlung der ADHS zugelassenes Arzneimittel (MPH oder ATX). Bei etwa 75\% dieser Patienten erfolgte die Erstverordnung durch einen Facharzt für Erkrankungen des ZNS, etwa 25\% der Erstverordnungen erfolgte durch Hausärzte ( $\triangleright$ Tab. 2). 
Tab. 2 Arztgruppen, die bei den Patienten $(n=1722)$ mit neu diagnostizierter ADHS im Zeitraum des darauffolgenden Jahres ein ADHSMedikament (MPH oder ATX) verordnet haben.

\begin{tabular}{|l|c|c|}
\hline Fachgruppe & $\begin{array}{l}\text { Anzahl } \\
\text { Patienten }\end{array}$ & $\begin{array}{l}\text { Anteil } \\
\text { Patienten (\%) }\end{array}$ \\
\hline Nervenärzte & 715 & 41,52 \\
\hline Allgemeinärzte Land & 283 & 16,43 \\
\hline Kinder- und Jugendpsychiater & 233 & 13,53 \\
\hline Psychiater & 200 & 11,61 \\
\hline Allgemeinärzte Stadt & 92 & 5,34 \\
\hline Internisten - hausärztlich & 73 & 4,24 \\
\hline Neurologen & 66 & 3,83 \\
\hline psychotherapeutisch tätige Ärzte & 39 & 2,26 \\
\hline Kinderärzte & 12 & 0,70 \\
\hline Internisten - Kardiologen & 4 & 0,23 \\
\hline Internisten - sonstige & 2 & 0,12 \\
\hline Internisten - Gastroenterologen & 1 & 0,06 \\
\hline Internisten - Hämatologen/ & 1 & 0,06 \\
\hline Onkologen & & 0,06 \\
\hline Urologen & 1 & \\
\hline
\end{tabular}

Von den 4 Quartalen nach Diagnosestellung wurden bei rund $31 \%$ der Patienten nur in einem Quartal ein Arzneimittel verordnet, bei $21 \%$ in 2 Quartalen, bei $22 \%$ in 3 Quartalen und bei $26 \%$, also in nur etwa einem Viertel der Fälle, in allen 4 Quartalen nach der initialen Diagnose.

Die Schwankungsbreite der Anzahl der Verordnungen für ADHS-Arzneimittel für den Betrachtungszeitraum (4 Quartale) nach Diagnosestellung war erheblich: Es erfolgten zwischen 1 und 47 Verordnungen (Mittelwert: 6, Median: 4). Entsprechend groß war auch die Variationsbreite hinsichtlich der Wirkstoffmengen: Bezogen auf die verordneten „defined daily doses“ (eine DDD für Erwachsene entspricht $40 \mathrm{mg} \mathrm{MPH}$ bzw. $80 \mathrm{mg}$ ATX) ergaben sich Mengen zwischen 2 und 1500 DDDs im Jahr (Mittelwert: 154, Median: 100). Verordnet wurde fast ausschließlich MPH, auf ATX entfielen weniger als $2 \%$ der Verordnungen.

\section{Unterkohorte „Patienten mit fortlaufender Erkrankung“}

Bei 6088 Patienten aus der Grundkohorte wurde die ADHS-Diagnose auch im Beobachtungszeitraum Quartal 4/2012 bis 3/ 2013 zumindest einmal pro Halbjahr bestätigt und somit als „fortlaufend dokumentierte Erkrankung“ definiert ( $>$ Abb. 1). Diese Patienten (61\% männlich, 39\% weiblich) waren überwiegend (zu 68\%) in der Altersklasse bis 35 Jahre ( Tab. 3). Bei den Frauen gab es weiterhin ein auffällig erhöhtes Vorkommen der ADHS-Diagnose in der Altersklasse 41 - 59 Jahre.

Von diesen Patienten erhielten 2295 (38\%) Medikamente, die für die Behandlung der ADHS im Erwachsenenalter zugelassen waren (MPH: 2224 Patienten, ATX: 39 Patienten, beide in Kombination: 32 Patienten), 1319 (22\%) andere Psychopharmaka und 2474 (41\%) keine Medikation ( $>$ Abb. 1). Der Anteil der Patienten, die keine Medikation erhielten, war unter den Männern etwas höher als unter den Frauen (43\% vs. 37\%).

Hinsichtlich der verordneten Mengen an ADHS-Arzneimitteln ergab sich ein breiter Streubereich ( $\triangleright$ Tab.4). Unter Berücksichtigung der Patienten mit mindestens einer Verordnung je Halbjahr in den 4 Quartalen des Beobachtungszeitraums wurden im Median 238 DDDs MPH bzw. 109 DDDs ATX bei den Männern, sowie 218 DDDs MPH bzw. 154 DDDs ATX bei den Frauen verordnet. Allerdings wurde auch eine kleine Gruppe von Patienten mit sehr hohen MPH-Verordnungsmengen (Maximalwerte > 1000 DDDs) identifiziert.

$\checkmark$ Tab.3 Altersverteilung der ADHS-Patienten mit fortlaufender Erkrankung separat dargestellt nach Geschlecht und Alter.

\begin{tabular}{|c|c|c|c|c|c|c|}
\hline $\begin{array}{l}\text { Altersklasse } \\
\text { (Jahre) }\end{array}$ & Anzahl Männer & Anteil Männer (\%) & Anzahl Frauen & Anteil Frauen (\%) & Anzahl gesamt & Anteil gesamt (\%) \\
\hline $19-20$ & 1025 & 27,4 & 418 & 17,8 & 1443 & 23,7 \\
\hline $21-25$ & 1121 & 30,9 & 445 & 19 & 1566 & 25,7 \\
\hline $26-30$ & 432 & 11,6 & 228 & 9,7 & 660 & 10,8 \\
\hline $31-35$ & 287 & 7,7 & 214 & 9,1 & 501 & 8,2 \\
\hline $36-40$ & 201 & 5,4 & 196 & 8,4 & 397 & 6,5 \\
\hline $41-45$ & 224 & 6 & 271 & 11,5 & 495 & 8,1 \\
\hline $46-50$ & 191 & 5,1 & 252 & 10,7 & 443 & 7,3 \\
\hline $51-59$ & 174 & 4,7 & 227 & 9,7 & 401 & 6,6 \\
\hline $60-69$ & 56 & 1,5 & 55 & 2,3 & 111 & 1,8 \\
\hline $70-91$ & 29 & 0,8 & 42 & 1,8 & 71 & 1,2 \\
\hline Summe & 3740 & 100 & 2348 & 100 & 6088 & 100 \\
\hline
\end{tabular}


- Tab. 4 Verteilung der verordneten definierten Tagesdosen (DDD, Defined Daily Doses) getrennt nach Geschlecht und Verordnungen je Halbjahr.

\begin{tabular}{|c|c|c|c|c|c|c|}
\hline & Gruppe & $\begin{array}{l}\text { mind. } 1 \text { vo je } \\
\text { Betrachtungshalbjahr }\end{array}$ & $\begin{array}{l}\text { DDD } \\
\text { Maximum }\end{array}$ & $\begin{array}{l}\text { DDD } \\
\text { Mean }\end{array}$ & $\begin{array}{l}\text { DDD } \\
\text { Median }\end{array}$ & $\begin{array}{l}\text { DDD } \\
\text { Minimum }\end{array}$ \\
\hline \multirow{4}{*}{$\begin{array}{l}\text { ADHS AM nicht } \\
\text { in beiden Halb- } \\
\text { jahren }\end{array}$} & \multirow{2}{*}{ Methylphenidat } & $\mathrm{m}$ & 650,00 & 77,92 & 50,00 & 6,25 \\
\hline & & w & 350,00 & 63,55 & 37,50 & 6,25 \\
\hline & \multirow{2}{*}{ Atomoxetin } & $\mathrm{m}$ & 224,00 & 56,99 & 28,00 & 3,50 \\
\hline & & w & 126,00 & 32,06 & 21,00 & 1,58 \\
\hline \multirow{4}{*}{$\begin{array}{l}\text { ADHS AM in } \\
\text { beiden Halbjahren }\end{array}$} & \multirow{2}{*}{ Methylphenidat } & $\mathrm{m}$ & 4354,16 & 317,71 & 237,50 & 8,33 \\
\hline & & w & 2824,31 & 277,45 & 218,00 & 18,75 \\
\hline & \multirow{2}{*}{ Atomoxetin } & $\mathrm{m}$ & 336,00 & 122,21 & 108,50 & 0,88 \\
\hline & & w & 294,00 & 152,67 & 154,00 & 11,20 \\
\hline
\end{tabular}

Grundsätzlich erhielten ADHS-Patienten häufiger Arzneimittel aus den Gruppen der Antidepressiva, Antipsychotika und Anxiolytika als Patienten der Vergleichskohorte ohne ADHS. Etwa $23 \%$ der Patienten mit fortlaufender Erkrankung, die ADHS-Medikamente erhielten, bekamen zusätzlich Antidepressiva (Frauen: $25 \%$, Männer: 15\%), ca. 5\% erhielten zusätzlich Antipsychotika und ca. 2\% Anxiolytika.

Bei Patienten, die kein ADHS-Medikament erhielten, wurden in $19 \%$ der Fälle Antidepressiva verordnet. Die Verordnung von Antipsychotika (7\%) und Anxiolytika (2\%) spielte eine eher untergeordnete Rolle. Im Gegensatz dazu erhielten nur knapp 7\% der Patienten aus der Vergleichskohorte Antidepressiva, ca. 2\% erhielten Antipsychotika und unter $1 \%$ erhielten Anxiolytika.

Darüber hinaus erhielten ADHS-Patienten ebenfalls deutlich häufiger Psychotherapieleistungen als Patienten der Vergleichskohorte. Jeder zweite ADHS-Patient erhielt Psychotherapie, wohingegen es in der Vergleichskohorte etwa jeder Fünfte war. Der Anteil der Frauen mit ADHS, die Psychotherapie erhielten, lag mit $62 \%$ höher als der Anteil der Männer mit $43 \%$.

Die Häufigkeit der Inanspruchnahme von Psychotherapieleistungen lag in der Gruppe der ADHS-Patienten mit ADHSspezifischer Medikation auf einem ähnlichen Niveau (54\%) wie in der Gruppe der Patienten ohne ADHS-Medikamente (48\%).

\section{Diskussion}

Die anonymisierten Daten von Kassenärztlichen Vereinigungen können wesentliche Informationen über das Zustandsbild der Versorgungssituation in Deutschland und damit zu Verbesserungsmöglichkeiten hinsichtlich Struktur und Qualität der Versorgung von psychiatrischen Erkrankungen wie ADHS liefern, die aufgrund der geringen Anzahl veröffentlichter Studien gerade für den Erwachsenenbereich dringend benötigt werden. Weiterhin ermöglichen sie wesentliche und für die klinische Praxis relevante Kernbefunde und Schlussfolgerungen hinsichtlich der Diagnoseprävalenz, der Rolle der Haus- und Fachärzte, der Häufigkeit und Varianz insbesondere medikamentöser Therapien sowie hinsichtlich geschlechtsspezifischer Unterschiede.

Die jüngst erschienene deutschsprachige S3-Leitline zur Aufmerksamkeitsdefizit-/Hyperaktivitätsstörung im Kindes-,
Jugend- und Erwachsenenalter [6] spiegelt wider, dass das Erkrankungsbild mittlerweile seinen Stellenwert in der Fachwelt gefunden hat und betroffene Patienten jeden Alters auch in Deutschland evidenzbasiert versorgt werden können. Neben initialen psychoedukativen Maßnahmen rückt nun verstärkt die Pharmakotherapie der ADHS in den Vordergrund. Bei Erwachsenen soll sie, je nach Präferenz des Patienten, bereits bei leichter Symptomausprägung zum Einsatz kommen können. Betroffenen wird hierdurch verdeutlicht, dass die erlebten Symptome behandelt werden können und bei vorhandenem Leidensdruck auch behandelt werden sollten.

Die Diagnoseprävalenz lag in der hier analysierten Stichprobe mit lediglich $0,1 \%$ unerwartet niedrig. Eine Auswertung von Routinedaten der Allgemeinen Ortskrankenkassen (AOK) zur Häufigkeit von ADHS-Diagnosen bei Erwachsenen für das Jahr 2014 kommt zu einem ähnlichen Ergebnis (0,3\%) [10]. Ausgehend von einer ADHS-Prävalenz bei Erwachsenen von ca. 3 \% [2] scheint nur ein Bruchteil dieser Patienten tatsächlich im Gesundheitssystem „anzukommen“. Dies steht in deutlichem Gegensatz zu der häufig in der Laienpresse propagierten Behauptung einer „Überdiagnostizierung“ von ADHS. Die möglichen Gründe für eine derart niedrige Diagnoseprävalenz sind vielfältig: Einerseits kommen Fehldiagnosen in Betracht, insbesondere, da eine ADHS im Erwachsenenalter häufig mit komorbiden Störungen wie Depression, Angst- und Persönlichkeitsstörungen einhergeht $[10,11]$. Weiterhin kann die nicht ausreichende Verfügbarkeit von Fachärzten in ländlichen Regionen sowie die immer noch in Teilen der Erwachsenenpsychiatrie vorhandene Skepsis, sich diesem Störungsbild zuzuwenden, zu einer Unterversorgung von Erwachsenen mit ADHS führen. Auch mag der Umstand, dass erst seit Sommer 2011 eine zugelassene Arzneimitteltherapie mit dem Wirkstoff Methylphenidat für die Behandlung der ADHS im Erwachsenenalter zur Verfügung stand, hemmend auf die Diagnosestellung eingewirkt haben. Die Daten der vorliegenden Studie können für eine Beantwortung dieser Frage jedoch keine genaueren Hinweise liefern. Unabhängig davon bleibt aber festzuhalten, dass offensichtlich erhebliche Defizite in der Versorgungssituation erwachsener Patienten mit ADHS bestehen [10]. 
Bei der Erstdiagnose spielen Hausärzte offenbar eine wichtige Rolle. Wie bei vielen anderen psychischen Erkrankungen übt auch bei ADHS im Erwachsenenalter der Hausarzt eine wesentliche Funktion als erste „Anlaufstelle“ aus [12].

Jedoch suchte nur etwa ein Drittel der Patienten, die beim Hausarzt mit ADHS diagnostiziert wurden, innerhalb eines Jahres zusätzlich einen Facharzt für Erkrankungen des ZNS auf. Bei diesen Patienten wurden dann jedoch weitere bzw. andere psychiatrische Störungen durch den Facharzt festgestellt. Dies unterstreicht die dringende Notwendigkeit zusätzlicher Fortbildungsangebote für den hausärztlichen Bereich sowie einer engen Zusammenarbeit zwischen Haus- und Fachärzten vor und nach der Diagnosestellung.

Möglicherweise könnte auch die Einführung neuer diagnostischer Instrumente sehr hilfreich sein [13]

Eine medikamentöse Therapie erhalten lediglich etwa $30 \%$ der neu diagnostizierten erwachsenen ADHS-Patienten. Hier steht zu befürchten, dass erwachsene ADHS-Patienten möglicherweise unzureichend behandelt werden trotz der nachgewiesenen guten Wirksamkeit und Sicherheit einer Pharmakotherapie $[14,15]$. Auffällig ist die enorme Schwankungsbreite hinsichtlich der Verordnungsmengen. Hierin könnte sich eine gewisse Unsicherheit der Behandler speziell im Umgang mit dieser Patientengruppe widerspiegeln. Sowohl Ärzte als auch Patienten mögen in ihrer Einstellung gegenüber der Symptomatik, gegenüber der Erkrankung und auch gegenüber der medikamentösen Therapie, die zumeist mit Methylphenidat erfolgt (einem Stimulans, das dem Betäubungsmittelgesetz unterliegt) unentschlossen und unzureichend informiert sein. Auch Patienten, die fortlaufend eine ADHS-Diagnose hatten, erhielten in nur $40 \%$ der Fälle eine medikamentöse Therapie. Der Befund, dass häufig zusätzliche Psychopharmaka wie z.B. Antidepressiva verschrieben wurden, lässt sich durch die häufig vorkommenden, komorbiden affektiven Erkrankungen erklären.

Bemerkenswert ist weiterhin, dass offenbar nur ein geringer Teil der Patienten nach einer Verordnung eines ADHS-Arzneimittels dieses auch regelmäßig zumindest über ein Jahr nach der Erstverordnung einnimmt. Dies deutet auf erhebliche Adhärenzdefizite bei der Arzneimitteltherapie hin. Eine intensive Aufklärung der Patienten vor sowie die regelmäßige Beratung während der Therapie erscheint auf Basis dieser Befunde notwendig. Positiv ist zu bewerten, dass ADHS-Patienten zusätzlich zu einer Pharmakotherapie häufig auch Psychotherapie erhalten. Hier scheint das in den Leitlinien geforderte „multimodale" Therapiekonzept schon weitgehend in die Versorgungsrealität übertragen worden zu sein.

Wie alle Studien mit Routinedaten der Krankenversorgung weist auch diese Studie spezifische Limitationen auf: Bei Leistungen und Diagnosen, die nicht abrechnungsrelevant sind, kann es zu Unterschätzungen kommen. In den Verordnungsdaten sind ausschließlich Rezepte enthalten, die der Patient auch tatsächlich bei einer Apotheke eingelöst hat. Somit ist nicht auszuschließen, dass die Anzahl der Verordnungen durch Ärzte unterschätzt wird, da nicht alle verordneten Medikamente tatsächlich abgeholt werden. Im Beobachtungszeitraum nahmen in etwa 1,3 Millionen Versicherte an Selektivverträgen in Bayern teil. Die im Rahmen dieser Selektivverträge erbrachten Leistungen werden nicht über die KV Bayerns abgerechnet. Somit ist mit einer zahlenmäßigen Unterschätzung der Leistungen und Diagnosen zu rechnen. Die den Patienten im Rahmen von Selektivverträgen verordneten Arzneimittel hingegen sind in den ausgewerteten Apothekenabrechnungsdaten mitenthalten. Es liegen keine Anhaltspunkte dafür vor, dass dies zu einer Verzerrung der Ergebnisse bezüglich Qualität und Struktur der Versorgung führte. Trotz der genannten Limitationen dürfte unsere Studie, die rund $85 \%$ aller gesetzlich Versicherten kassenübergreifend in Bayern abdeckt, ein sehr umfassendes Bild der Versorgungsrealität erwachsener Patienten mit ADHS liefern.

\section{KONSEQUENZEN FÜR KLINIK UND PRAXIS}

- Die Prävalenz der ADHS im Erwachsenenalter in Bayern im Jahr 2012 lag in dieser Studie mit 0,1 \% erheblich unter dem in der internationalen Literatur angegebenen Wert von ca. $3 \%$.

- Hausärzte sind eine wesentliche Anlaufstelle für erwachsene Patienten mit einer ADHS. Dies unterstreicht die Notwendigkeit einer engen Zusammenarbeit zwischen Hausärzten und Spezialisten für Erkrankungen des ZNS.

- Um die insgesamt unzureichende Medikamentenadhärenz der Patienten mit ADHS zu verbessern, sollte eine enge Betreuung der Patienten vor und nach der Arzneimittelverordnung erfolgen.

\section{Danksagung}

Der Beitrag von Johannes Thome zu diesem Projekt wurde auch unterstützt durch das Horizon-2020-Forschungs- und Innovationsprogramm der Europäischen Union unter der Fördernummer 667302 (CoCA).

Die Publikation reflektiert ausschließlich den Standpunkt der Autoren, und die Kommission ist nicht verantwortlich für jedweden Gebrauch, der von der darin enthaltenen Information gemacht werden könnte.

Interessenkonflikt

Johannes Thome und Joerg Hasford haben von der KVB finanzielle Unterstützung für die eingereichte Arbeit erhalten. Johannes Thome hat darüber hinaus finanzielle Unterstützung (z. B. Vortragshonorare, Stipendien für Forschungsprojekte und wissenschaftliche Veranstaltungen, Mitgliedschaft in Beratungsgremien) erhalten von Actelion, AstraZeneca, Bristol-Meyers Squibb, Ever Neuro Pharma, Janssen-Cilag, Lilly, Lundbeck, Medice, Merz, Novartis, Pfizer, Roche, Servier, Shire, von denen einige Medikamente zur Behandlung der ADHS im Erwachsenenalter herstellen. Kerstin Behnke, Lydia Steer und Martina Weiher sind Mitarbeiterinnen der KV Bayerns. Markus Gleitz arbeitet hauptberuflich bei Medice. Die Kosten für die Open-Access-Veröffentlichung wurden von Medice getragen. 


\section{Literatur}

[1] Kooij SJ, Bijlenga D, Salerno L et al. Updated European consensus statement on diagnosis and treatment of adult ADHD. Eur Psychiatr 2019; $56: 14-34$

[2] Lam AP, Müller HHO, Philipsen A. ADHS im Erwachsenenalter - Diagnostik und Therapie. Fortschr Neurol Psychiatr 2017; 85: 696-707

[3] Mohr Jensen C, Steinhausen HC. Time trends in incidence rates of diagnosed attention-deficit/hyperactivity disorder across 16 years in a nationwide Danish registry study. J Clin Psychiatry 2015; 76: 334 341

[4] Philipsen A, Tebartz van Elst L, Lesch KP et al. Wirksamkeit und Wirkmechanismen von Psychotherapie bei der Behandlung der Aufmerksamkeitsdefizit-/Hyperaktivitätsstörung (ADHS) bei Kindern und Erwachsenen. Psychother Psych Med 2009; 59: 132 - 140

[5] NICE - National Institute for Health and Clinical Excellence. Attention deficit hyperactivity disorder: diagnosis and management. NICE guideline [NG87], March 2018

[6] AWMF (Arbeitsgemeinschaft der Wissenschaftlichen Medizinischen Fachgesellschaften). Langfassung der interdisziplinären evidenz- und konsensbasierten (S3) Leitlinie „Aufmerksamkeitsdefizit-/Hyperaktivitätsstörung (ADHS) im Kindes-, Jugend- und Erwachsenenalter“. AWMF Registriernummer 028-045, 2018

[7] Ramos-Quiroga JA, Montoya A, Kutzelnigg A et al. Attention deficit hyperactivity disorder in the European adult population: prevalence, disease awareness, and treatment guidelines. Curr Med Res Opin 2013; 29: 1 - 12
[8] Polyzoi M, Ahnemark E, Medin E et al. Estimated prevalence and incidence of diagnosed ADHD and health care utilization in adults in Sweden - a longitudinal population-based register study. Neuropsychiatr Dis Treat 2018; 14: 1149-1161

[9] Madsen KB, Ersbøll AK, Olsen J et al. Geographic analysis of the variation in the incidence of ADHD in a country with free access to healthcare: a Danish cohort study. Int J Health Geogr 2015; 14: 24

[10] Bachmann C], Philipsen A, Hoffmann F. ADHS in Deutschland: Trends in Diagnose und Medikamentöser Therapie. Deutsches Ärzteblatt 2017: 114: 141 - 148

[11] Rösler M, Retz W. Diagnose, Differentialdiagnose und komorbide Leiden der Aufmerksamkeitsdefizit-/Hyperaktivitätsstörung (ADHS) im Erwachsenenalter. Psychotherapie 2008; 13: 175-183

[12] Schulze G. ADHS beim Erwachsenen: Diagnostik, Therapie und hausärztliche Möglichkeiten. Z Allg Med 2002; 78: 361 - 366

[13] Grabemann M, Zimmermann M, Strunz L et al. Neue Wege in der Diagnostik der ADHS bei Erwachsenen. Psychiat Prax 2017; 44: 221 227

[14] Retz W, Retz-Junginger P, Thome J et al. Pharmacological treatment of adult ADHD in Europe. World J Biol Psychiatry 2011; 12(Suppl. 01): $89-94$

[15] Philipsen A, Jans T, Graf E et al. Effects of Group Psychotherapy, Individual Counseling, Methylphenidate, and Placebo in the Treatment of Adult Attention-Deficit/Hyperactivity Disorder. A Randomized Clinical Trial. JAMA Psychiatry 2015; 72: 1199-1210 